\title{
GIẢI PHÁP CHO VẤN ĐỀ BIẾN ĐỔI KHÍ HậU VÀ NĂNG LỰ̛̣G TRONG THẾ KỶ 21
}

Công Thuỳ Dương, Đoàn Thu Dung, Phan Thuỳ Dương, Nguyễn Tấn Dũng, Hoàng Thị Hồng Chúc, Hoàng Thị Linh Chi, Vũ Quỳnh Chi, Nguyễn Hoàng Chi, Nguyễn Cẩm Dương, Lê Thị Thanh Diễm.

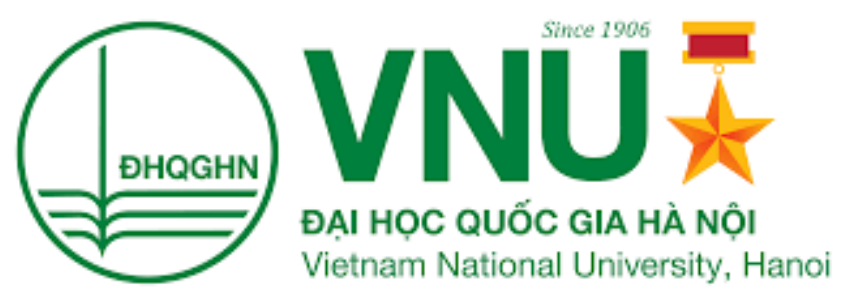

Đại học Quốc gia Hà Nội, Việt Nam.

Hà Nội, ngày 09 tháng 01 năm 2022

Preprint DOI:10.31219/osf.io/wnbmh

Bảo vệ môi trường sống xanh - sạch - đẹp là vấn đề đang được cả thế giới quan tâm. Có rất nhiều hội nghị tầm cỡ toàn cầu hoặc khu vực đã được tổ chức để bàn bạc và tìm ra hướng giải quyết vấn đề đó (Khuc, 2022; Q.-H. Vuong, 2021). Trong đó, sự biến đổi khí hậu, cạn kiệt nguồn năng lượng và hiệu ứng nhà kính là những vấn đề nóng hổi, đây là một trong những thách thức lớn đối với toàn nhân loại vì chúng đang ảnh hưởng trực tiếp đến sinh thái, môi trường và cuộc sống của con người.

Việc cháy rừng tại Hy Lạp hồi tháng 8/2021 được cho là một trong số những sự kiện nóng bỏng về vấn đề này. Theo Thủ tướng Hy Lạp Kyriakos Mitsotakis, đây là thảm họa môi trường tồi tệ nhất của đất nước. Từ ngày 3 tháng 8 đến ngày 12 tháng 8 , trận hỏa hoạn lớn nhất trên đảo Evia (hòn đảo lớn thứ hai của đất nước) đã tàn phá phần lớn phần phía bắc của hòn đảo. Đám cháy đã cướp đi sinh mạng của 3 người và phá hủy gần 100.000 ha rừng và đất nông nghiệp (Huong, 2021). Theo Tổ chức Khí tượng Thế giới (WMO), cháy rừng là một phần của xu hướng thời tiết khắc nghiệt xảy ra do biến đổi khí hậu do con người gây ra. 


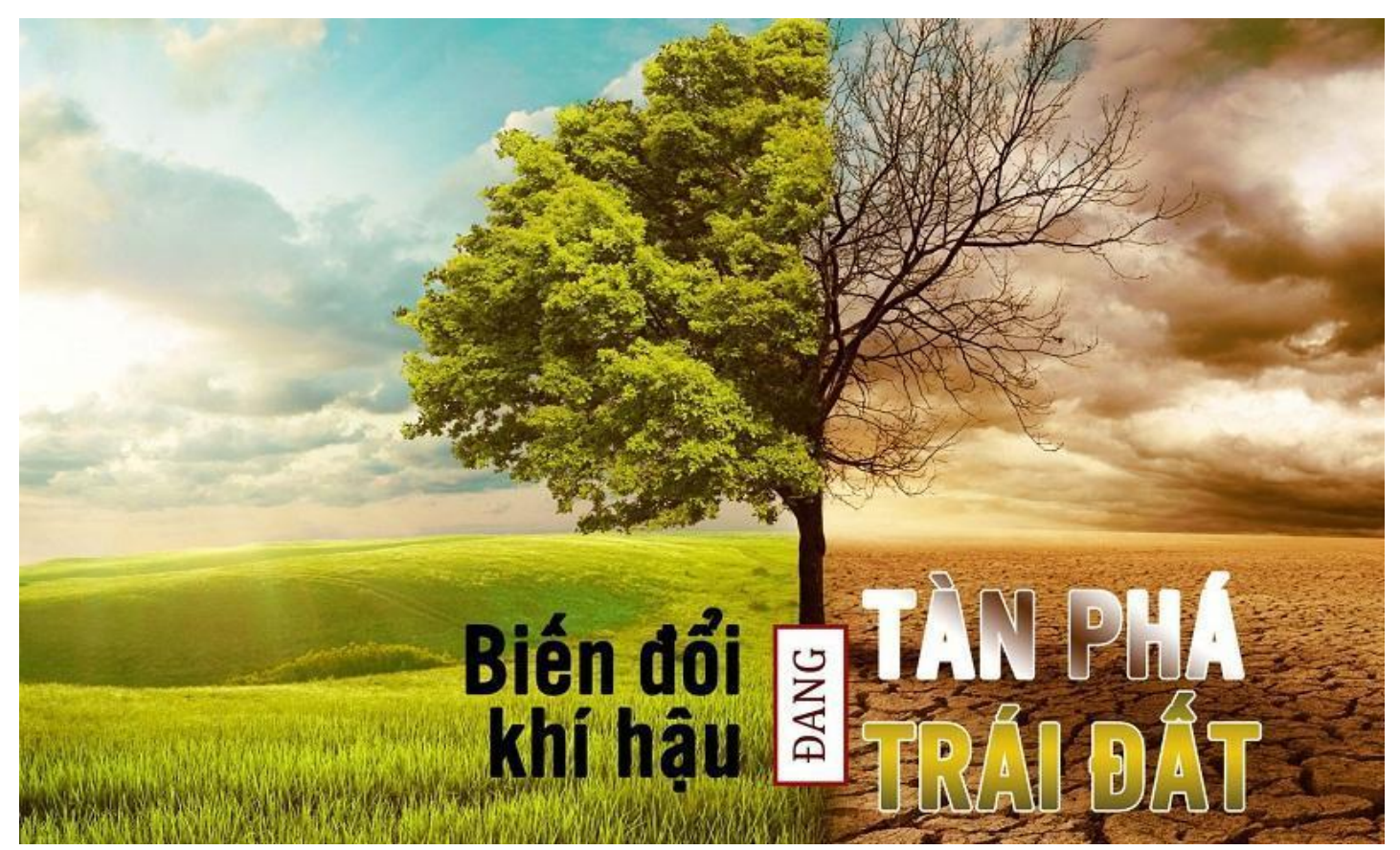

Hình 1. Hình ảnh minh họa cho việc biến đổi khi hậu đang tàn phá trái đất Nguồn: Internet

\section{https://khbvptr.vn/bien-doi-khi-hau-la-gi/}

Biến đổi khí hậu, ngoài việc gây ra một số tác động, còn gây ra nhiều mối đe dọa đối với quyền con người. Biến đổi khí hậu đang tác động ngày càng rõ nét đến con người, với những đợt thiên tai, hạn hán, lũ lụt kéo dài gây mất mùa, thiếu lương thực; ô nhiễm môi trường đất, nước, không khí... ảnh hưởng trực tiếp đến sức khỏe con người (H. T. H. Hanh, 2022; N. T. Hanh, 2022; Van Khuc et al., 2020; Q. Vuong et al., 2021); sụt lún, sạt lở đất khiến người dân mất nhà cửa, thậm chí tính mạng. Những việc làm này đã trực tiếp xâm hại đến quyền sống của con người (Huyền, 2016).

Nhưng Việt Nam và thế giới vẫn đang nỗ lực để đối mặt với sự biến đổi khí hậu. Trong hội nghị COP26, Việt Nam đã đưa ra một số cam kết cũng như một số hành động hiệu quả và mang tính xây dựng trong chống biến đổi khí hậu toàn cầu. Việt Nam cũng đã công bố các kế hoạch hành động và sáng kiến để giải quyết vấn đề biến đổi khí hậu, bao gồm Chiến lược quốc gia về biến đổi khí hậu, Kế hoạch thực hiện Thỏa thuận Paris và Đề án quản lý khí thải. phát thải nhà kính và Kế hoạch quốc gia về thích ứng với biến đổi khí hậu giai đoạn 2021-2030, mục tiêu đến năm 2050. Nó cũng quy định các vấn đề liên quan đến ứng phó với biến đổi khí hậu, chẳng hạn như luật về phòng chống thiên tai, luật về bảo vệ môi trường, pháp luật về tài nguyên nước, v.v. (Nhâm, 2021). 
Thế giới đề cập đến 7 sáng kiến nhằm giảm thiểu biến đổi khí hậu: đầu tư mạnh tay cho năng lượng sạch; Mỹ - Trung hành động chống biến đổi khí hậu; tuần lễ khí hậu châu Phi tạo cú hích cho hành động khu vực; nước chủ nhà COP26 "nhắc khéo" các nhà tài trợ; chuyển đổi hệ thống lương thực toàn cầu; hành động của tuổi trẻ về biến đổi khí hậu; hội nghị tiền COP26 (Thanh, 2021). Việt Nam cùng thế giới đã, đang và sẽ luôn nỗ lực trong việc giảm thiểu tác động của biến đổi khí hậu đối với đời sống con người (Khuc, 2022).

Bên cạnh sự biến đổi khí hậu, vấn đề năng lượng đang dần cạn kiệt cũng đang ở mức đáng báo động không còn là nỗi lo ở tương lai xa, tình trạng cạn kiệt nguồn năng lượng đang dần được chứng minh bằng những sự kiện diễn ra trong thực tế. Vào đầu năm nay, mùa đông ở Châu Âu kéo dài và lạnh bất thường làm cạn kiệt nguồn dự trữ khí đốt, do ảnh hưởng của một loạt những cơn bão, ở vùng Vịnh các nhà máy lọc dầu buộc phải đóng cửa. Tiêu biểu là sự phục hồi kinh tế sau đại dịch COVID-19 dẫn tới nhu cầu năng lượng tăng cao (B. Anh, 2021). Việt Nam xuất hiện sự mất cân đối nghiêm trọng giữa phát triển điện năng và phát triển kinh tế, bắt nguồn từ việc tổn thất và lãng phí nguồn năng lượng nhiều, hiệu quả sử dụng điện năng còn thấp.
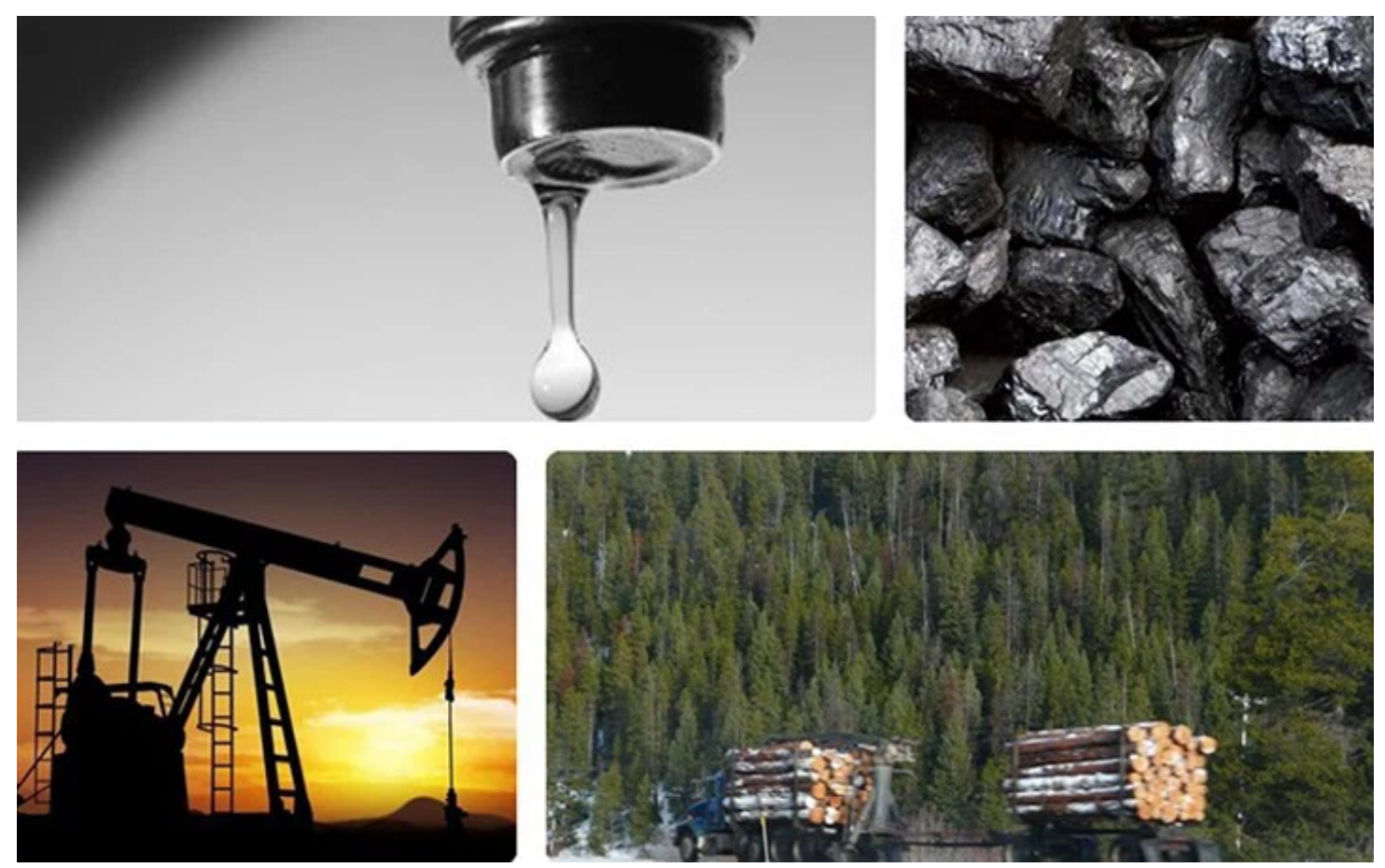

Hình 2. Hình ảnh minh họa cho sụ cạn kiệt các nguồn năng luợng trên thế giới Nguồn: Internet

https://cafef.vn/nhung-tai-nguyen-thien-nhien-hang-dau-se-som-can-kiet-20180 920140208993.chn 
Vào những năm 70 của thế kỷ XX, để đối phó với vấn đề cạn kiệt nguồn năng lượng hóa thạch, nhiều quốc gia nhắm đến việc sử dụng năng lượng hạt nhân làm lựa chọn thay thế. Tuy nhiên, chỉ khoảng 10 năm sau, vào những năm 80 , thế giới đã chứng kiến sự thất bại của kế hoạch này. Nguyên nhân nằm ở vấn đề an toàn của các nhà máy điện hạt nhân còn nhiều điểm hạn chế: rò rỉ phóng xạ, tai nạn hạt nhân, con người bị nhiễm phóng xạ,...(Trần, 2007).

Tuy nhiên, hiện nay chúng ta đã có nhiều chính sách hợp lý để cải thiện tình hình. Tại Việt Nam, ngày 11 tháng 2 năm 2020, Bộ Chính trị đã ban hành Nghị quyết số $55-\mathrm{NQ} / \mathrm{TW}$ về định hướng Chiến lược phát triển năng lượng quốc gia của nước ta đến năm 2030, tầm nhìn đến năm 2045. Theo một số đánh giá, khoảng 16 triệu tấn dầu quy đổi tương đương với khoảng 103,7 tỷ $\mathrm{kWh}$ điện sẽ được tiết kiệm so với nhu cầu năng lượng phục vụ phát triển kinh tế xã hội nếu thực hiện Chương trình mục tiêu này (Bình, 2021). Tính tới tháng 7/2020, về nguồn điện năng lượng tái tạo, hệ thống điện quốc gia có tổng cộng 99 nhà máy điện mặt trời vận hành, trong đó, tổng công suất là $5.053 \mathrm{MW}$. Đồng thời, tổng công suất 11 nhà máy điện gió đang hoạt động là 429 $\mathrm{MW}$, trong đó gồm $325 \mathrm{MW}$ điện sinh khối cùng với gần $10 \mathrm{MW}$ điện chất thải rắn. Có thể thấy, tổng công suất điện gió và mặt trời đã là $5.482 \mathrm{MW}$, chiếm khoảng $9,5 \%$ tổng công suất nguồn đặt của hệ thống. Không dừng lại ở đó, về điện mặt trời mái nhà, đã có trên 47.000 hệ thống được lắp đặt với tổng công suất $1.128 \mathrm{MWp}$, tính đến 8/2020. (Hưng, 2020).

Trên thế giới, nhiều quốc gia đã đang đẩy mạnh sử dụng các nguồn năng lượng tái tạo. Có thể thấy, Côte d'Ivoire (thuộc Tây Phi) sản xuất khoảng $2.000 \mathrm{MW}$ điện mỗi năm, trong đó nhiệt điện chiếm $75 \%$ và phần còn lại là thủy điện. Bên cạnh đó, năng lượng tái tạo tăng tỷ lệ lên $11 \%$ trên tổng lượng điện vào năm 2020 và lên tới $16 \%$ vào năm 2030 là mục tiêu chính của nước này ( $\mathrm{M}$. Anh, 2019). Ngoài ra, ta không thể không kể đến Hội nghị thượng đỉnh Liên minh châu Âu về vấn đề năng lượng. Liên minh châu Âu (EU) đã hối thúc các quốc gia sử dụng khẩn cấp "hộp công cụ" để hỗ trợ cho những người tiêu dùng dễ bị tổn thương nhất cùng các công ty châu Âu trong ngắn hạn. (Quyên, 2021).

Ngoài hai vấn đề nóng nêu ở trên, hiệu ứng nhà kính cũng là một chủ đề vô cùng cấp thiết bởi nó là một trong những hậu quả nhức nhối nhất của biến đổi khí hậu toàn cầu. Khi nồng độ khí nhà kính tăng lên, nhiệt độ bề mặt trung bình toàn cầu cũng tăng theo. Do đó, các hiện tượng thời tiết khắc nghiệt đã trở nên phổ biến hơn trên khắp hành tinh. (Vào năm 2020, hạn hán nghiêm trọng đã tấn công nhiều khu vực khác nhau của lục địa Nam Mỹ, bao gồm cả miền bắc Argentina và Paraguay... Ngoài ra, khu vực Bắc Đại Tây Dương đã trải qua một mùa mưa bão dữ dội khác thường, với 
30 trận bão xoáy nhiệt đới, phá kỷ lục mùa mưa bão của năm 2005) (DWRM, 2020) . Nhiệt độ toàn cầu gia tăng cũng dẫn đến sự tan chảy của Greenland, Nam Cực và các sông băng trên khắp thế giới và khiến mực nước biển dâng cao. Trên toàn cầu, mực nước biển đã tăng trung bình 3,29 (+/- 0,3) mm mỗi năm, và đạt đỉnh vào năm 2020 . (N. R. Claire Ransom, 2020).

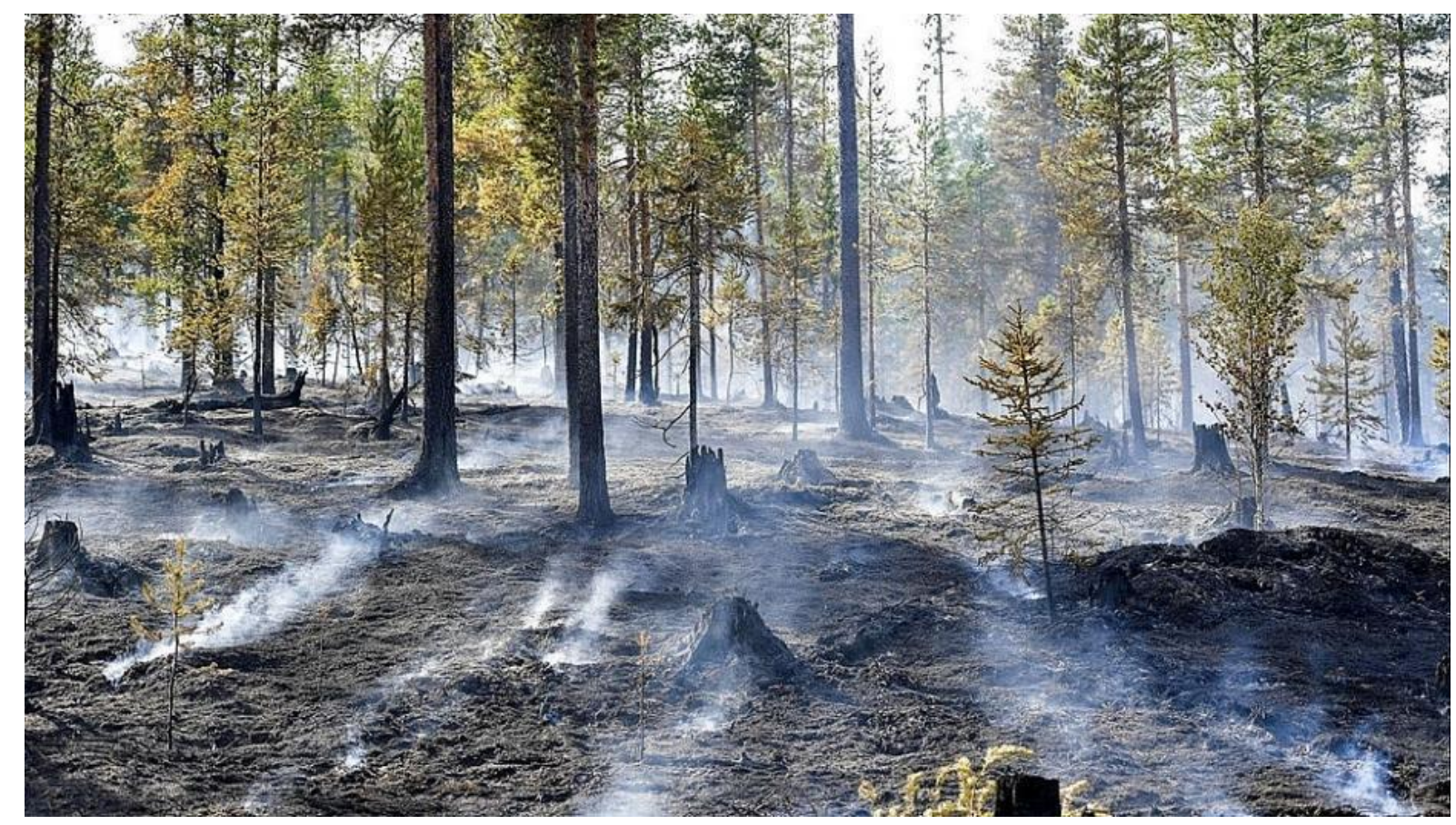

Hình 3. Hậu quả của hiệu ứng nhà kính

\section{Nguồn: Internet}

https://kinhtemoitruong.vn/hieu-ung-nha-kinh-va-nhung-hau-qua-kho-luong-8820.htm

Để ứng phó với tình trạng hiệu ứng nhà kính ngày càng báo động, các tổ chức về môi trường đã tích cực tham gia các hội nghị để đưa ra những thỏa thuận, giải pháp nhằm cải thiện hiện tượng khí hậu này. Tuy vậy không ít những nỗ lực đó đã thất bại. Hiệp định Paris diễn ra vào năm 2015 đã đề ra mục tiêu giữ cho nhiệt độ Trái Đất không vượt $1,5^{\circ} \mathrm{C}$, tuy nhiên nó không diễn ra thành công bởi các bên tham gia khó có thể hiện thực hóa mục tiêu ấy. Dựa trên nội dung của báo cáo thứ sáu (AR6) của Ủy ban liên chính phủ về biến đổi khí hậu (IPCC) đã nhấn mạnh rằng: "Một số thay đổi, tiêu biểu như việc hạn chế mực nước biển dâng cao là không thể thực hiện trong hàng trăm hoặc hàng nghìn năm". Theo các chuyên gia khí hậu và năng lượng đến từ Thụy Điển, $\mathrm{Na}$ Uy và Áo, việc đạt được mục tiêu hạn chế nhiệt độ Trái đất tăng hơn $1,5^{\circ} \mathrm{C}$ 
sẽ đòi hỏi phải giảm đáng kể mức sử dụng năng lượng, điều mà bất kỳ nền kinh tế nào trên thế giới cũng khó có khả năng chịu được (BBT, 2021).

Hiệu ứng nhà kính làm gia tăng các rủi ro hiện có và làm trầm trọng thêm các yếu tố có thể dẫn đến xung đột mất an ninh. Chẳng hạn, sự ảnh hưởng của hiệu ứng nhà kính đến nông nghiệp và chủ nghĩa mục vụ với các yếu tố chính trị dân tộc ở Sudan đã góp phần vào sự leo thang bạo lực căng thẳng ở Darfur và khiến việc giải quyết xung đột trở nên khó khăn hơn. Ở Syria và Iraq, tổ chức cực đoan Daesh khai thác sự cạnh tranh ngày càng gia tăng về tài nguyên thiên nhiên và nước được vũ khí hóa bằng cách kiểm soát việc tiếp cận và chuyển hướng các con sông. Ngoài ra, các hiện tượng thời tiết cực đoan ở Trung Mỹ và Caribe đã tàn phá cơ sở hạ tầng quan trọng và khiến người dân buộc phải di dời, và ở một số nơi thậm chí còn tác động đến tỷ lệ tội phạm khiến cho con số này tăng đột biến. (R. DiCarlo, 2020)

Ở quy mô toàn cầu, Liên Hiệp Quốc đã có nhiều cố gắng trong cuộc chiến chống lại hiệu ứng nhà kính. Một số những thành tựu đáng kể đã đạt được như Công ước Khung của Liên Hiệp Quốc về $\mathrm{BĐKH} \mathrm{(UNFCCC)} \mathrm{-} \mathrm{có} \mathrm{hiệu} \mathrm{lực} \mathrm{chính} \mathrm{thức} \mathrm{từ}$ ngày 19-3-1994 với mục tiêu đạt được sự ổn định khí nhà kính trong khí quyển ở mức có thể ngăn ngừa sự can thiệp nguy hiểm của con người đối với hệ thống khí hậu, Nghị định thư Kyoto $(\mathrm{KP})$ - có hiệu lực từ ngày 16-12-2005 nhằm hỗ trợ các nước đang phát triển thực hiện phát triển bền vững và các nước phát triển thực hiện cam kết giảm phát thải khí nhà kính định lượng (MET, 2018). Nhận thức rõ tác động của hiệu ứng nhà kính, Việt Nam cũng đã sớm tham gia các hoạt động ứng phó của khu vực và quốc tế về hiệu ứng nhà kính cũng như $\mathrm{BĐKH} \mathrm{như:} \mathrm{Tham} \mathrm{gia} \mathrm{kí} \mathrm{Công} \mathrm{ước} \mathrm{Khung} \mathrm{của}$ Liên Hiệp Quốc về BĐKH (UNFCCC); tham gia kí Nghị định thư Kyoto (KP); thông qua Luật sử dụng năng lượng tiết kiệm và hiệu quả...; triển khai các chương trình, đề án liên quan trực tiếp đến giảm nhẹ phát thải khí nhà kính: Chương trình mục tiêu quốc gia về sử dụng năng lượng tiết kiệm và hiệu quả; Chương trình hành động quốc gia về giảm phát thải khí nhà kính thông qua nỗ lực hạn chế mất rừng và suy thoái rừng, quản lý bền vững tài nguyên rừng,...(Vihema, 2020).

Tóm lại, sự biến đổi khí hậu, cạn kiệt nguồn năng lượng, và hiệu ứng nhà kính đang đe doạ đến môi trường cũng như cuộc sống của con người. Chính vì thế, một số giải pháp theo chúng tôi nên tập trung ưu tiên trong thời gian tới là: (1) tăng cường chuyển đổi văn hóa môi trường từ nhận thức, đến hành vi môi trường (Khuc, 2021; Van Khuc et al., 2021; Q. H. Vuong, 2021; Q. H. et al. Vuong, 2018), (2) sử dụng khoa học công nghệ, thông tin truyền thông để cải tiến chất lượng các giải pháp (Nguyen \& Vuong, 2021; Q. Vuong, 2020; Q. H. Vuong, 2018); (3) tăng cường hợp tác thu hút sự tham gia của lực lượng doanh nghiệp vào bảo vệ môi trường (Q.-H. 
Vuong, 2021; Q. H. Vuong et al., 2021) để có thể đối phó với những vấn đề trên, trả lại sự xanh, sạch, đẹp vốn có cho môi trường tự nhiên.

\section{Tài liệu tham khảo:}

Anh, B. (2021). Thế giới đói năng lượng. Tuổi Trẻ Online. https://tuoitre.vn/the-gioi-doi-nang-luong-20211012084245602.htm

Anh, M. (2019). Đẩy mạnh sủ dụng các nguồn năng lượng tái tạo. Báo Nhân Dân. https://nhandan.vn/tin-tuc-the-gioi/ay-manh-su-dung-cac-nguon-nang-luong-tai-tao-348461/

BBT. (2021). Thất bại trong việc đối phó với biến đổi khí hậu: Thảm họa không thể tránh khỏi trên Trái đất. Sputnik Việt Nam.

https://vn.sputniknews.com/20211101/that-bai-trong-viec-doi-pho-voi-bien-doi-khi-hau-tham-ho a-khong-the-tranh-khoi-tren-trai-dat-12329804.html

Bình, A. (2021). Sử dụng tiết kiệm năng luợng và hiệu quả là nền tảng cho phát triển bền vũng. Bộ Công Thương Việt Nam.

https://moit.gov.vn/tin-tuc/su-dung-nang-luong-tiet-kiem-va-hieu-qua/su-dung-tiet-kiem-nang-lu ong-va-hieu-qua-la-nen-tang-cho-phat-trien-ben-vung.html

DWRM. (2020). Năm 2020 trở thành một trong ba năm nóng nhất tùng được ghi nhận trên Trái Đất.

Bộ Tài Nguyen và Môi Trường.

http://dwrm.gov.vn/index.php?language=vi\&nv=news\&op=Nhin-ra-The-gioi/Nam-2020-tro-tha nh-mot-trong-ba-nam-nong-nhat-tung-duoc-ghi-nhan-tren-Trai-Dat-9600

Hanh, H. T. H. (2022). Nguyên nhân và giải pháp cho vấn đề ô nhiễm và cạn kiệt nguồn nước. $O S F$ Preprints. https://doi.org/10.31219/osf.io/ph2rs

Hanh, N. T. (2022). Thực trạng và giải pháp cho vấn đề ô nhiễm không khí ở Việt Nam. $O S F$ Preprints. https://doi.org/10.31219/osf.io/jrxmd

Hưng, G. (2020). Khai thác, sử dụng hiệu quả các nguồn năng lượng tái tạo. Báo Điện Tử Đảng Cộng Sản Việt Nam.

https://dangcongsan.vn/khoa-hoc-va-cong-nghe-voi-su-nghiep-cong-nghiep-hoa-hien-dai-hoa-da t-nuoc/diem-nhan-khoa-hoc-va-cong-nghe/khai-thac-su-dung-hieu-qua-cac-nguon-nang-luong-ta i-tao-564429.html

Huong, T. (2021). Hy Lạp đã khống chế được các đám cháy rừng kéo dài 9 ngày qua. Báo Tin Túcc. https://baotintuc.vn/the-gioi/hy-lap-da-khong-che-duoc-cac-dam-chay-rung-keo-dai-9-ngay-qua20210812103847052.htm

Huyền, Á. (2016). Đảm bảo quyền con nguoời trước tác động của biến đổi khi hậu. VOV. https://vovworld.vn/vi-VN/binh-luan/dam-bao-quyen-con-nguoi-truoc-tac-dong-cua-bien-doi-kh i-hau-447287.vov.

Khuc, Q. Van. (2021). Environmental culture thoughts to make a better world for our nature and children. OSF Preprints. https://doi.org/10.31219/osf.io/g5zex

Khuc, Q. Van. (2022). Về khả năng ứng dụng của hệ xử lý thông tin $3 \mathrm{D}$ và nguyên lý bán dẫn giá trị trong tìm kiếm giải pháp cho vấn đề ô nhiễm môi trường và biến đổi khí hậu ở Việt Nam. Tạp Chí Kinh Tế và Dư Báo, 1-5.

https://kinhtevadubao.vn/ve-kha-nang-ung-dung-cua-he-xu-ly-thong-tin-3d-va-nguyen-ly-ban-da n-gia-tri-trong-tim-kiem-giai-phap-cho-van-de-o-nhiem-moi-truong-va-bien-doi-khi-hau-o-vietnam-20840.html

MET. (2018). Tài liệu hướng dẫn dạy và học về Úng phó với biển đổi khi hậu. http://chinhsach.dmptc.gov.vn:8045/Uploads/Thu vien tai lieu/To chuc NGO/tài_liệu_dạy_và_học_về_Úng_phó_với_biến_dổi_khí_hậu.pdf

N. R. Claire Ransom, V. H. (2020). The State of the Global Climate 2020. World Meteorological Organization.

https://public.wmo.int/en/our-mandate/climate/wmo-statement-state-of-global-climate 
Nguyen, M.-H., \& Vuong, Q.-H. (2021). Evaluation of the Aichi Biodiversity Targets: the international collaboration trilemma in interdisciplinary research. Pacific Conservation Biology. https://doi.org/10.1071/pc21026

Nhâm, N. (2021). Việt Nam tham gia tích cực, trách nhiệm chống biến đổi khí hậu. Báo Điện Tư Đảng Cộng Sản Việt Nam.

https://dangcongsan.vn/the-gioi/nhung-van-de-toan-cau/viet-nam-tham-gia-tich-cuc-trach-nhiem -chong-bien-doi-khi-hau-596974.html

Quyên, T. (2021). Hội nghị thương đỉnh Liên minh châu Âu "nóng” với vấn đề năng lượng. Vietnam Plus.

https://www.vietnamplus.vn/hoi-nghi-thuong-dinh-lien-minh-chau-au-nong-voi-van-de-nang-luo ng/748308.vnp

R. DiCarlo. (2020). R. DiCarlo, "Climate change multiplying factors that lead to insecurity for millions, Rosemary DiCarlo tells 'Arria Formula' meeting. Political and Peacebuilding Affairs. https://dppa.un.org/en/climate-change-multiplying-factors-lead-to-insecurity-millions-rosemarydicarlo-tells-arria-formula.

Thanh, H. (2021). 7 nỗ lực chống biến đổi khí hậu nổi bật trước thềm Hội nghị COP26. Báo Tin Tức. https://baotintuc.vn/the-gioi/7-no-luc-chong-bien-doi-khi-hau-noi-bat-truoc-them-hoi-nghi-cop2 6-20211011155850756.htm

Trần, M. T. (2007). Năng lượng từ hyđrô sẽ thay thế dầu khí, nguyên tử. Báo Nhân Dân. https://nhandan.vn/khoa-hoc/nang-luong-tu-hydro-se-thay-the-dau-khi-nguyen-tu-434949

Van Khuc, Q., Pham, L., Tran, M., Nguyen, T., Tran, B. Q., Hoang, T., Ngo, T., \& Tran, T. D. (2021). Understanding vietnamese farmers' perception toward forest importance and perceived willingness-to-participate in redd+ program: A case study in nghe an province. Forests, 12(5), 1-14. https://doi.org/10.3390/f12050521

Van Khuc, Q., Phu, T. V., \& Luu, P. (2020). Dataset on the Hanoian suburbanites' perception and mitigation strategies towards air pollution. Data in Brief, 33, 106414. https://doi.org/10.1016/j.dib.2020.106414

Vihema. (2020). Đóng góp do quốc gia tụ quyết định của Việt Nam. https://vihema.gov.vn/wp-content/uploads/2020/10/NDC_VN_Clean_final.pdf

Vuong, Q.-H. (2021). Western monopoly of climate science is creating an eco-deficit culture. Economy, Land \& Climate Insight, 11, 1-9. https://elc-insight.org/western-monopoly-of-climat

Vuong, Q. (2020). From children's literature to sustainability science, and young scientists for a more sustainable Earth From children's literature to sustainability science, and young scientists for a more sustainable Earth. Journal of Sustainability Education, 24(December), 2019-2021.

Vuong, Q. H. (2018). The (ir)rational consideration of the cost of science in transition economies. Nature Human Behaviour, 2(1), 5. https://doi.org/10.1038/s41562-017-0281-4

Vuong, Q. H. (2021). The semiconducting principle of monetary and environmental values exchange. Economics and Business Letters, 10(3), 284-290. https://doi.org/10.17811/ebl.10.3.2021.284-290

Vuong, Q. H. et al. (2018). Cultural additivity: behavioural insights from the interaction of Confucianism, Buddhism and Taoism in folktales. Palgrave Communications, 4(143). https://doi.org/10.1057/s41599-018-0189-2

Vuong, Q. H., Ho, M. T., Nguyen, M. H., Pham, T. H., Vuong, T. T., Khuc, Q., Ho, H. A., \& La, V. P. (2021). On the environment-destructive probabilistic trends: A perceptual and behavioral study on video game players. Technology in Society, 65(February), 101530.

https://doi.org/10.1016/j.techsoc.2021.101530

Vuong, Q., Phu, T. V., Le, T. T., \& Khuc, Q. Van. (2021). Exploring Inner-City Residents' and Foreigners' Commitment. Data, 6(39). https://doi.org/10.17632/8sv4j2r9wk.2 